\title{
MiR-49I-5p, as a Tumor Suppressor, Prevents Migration and Invasion of Breast Cancer by Targeting ZNF-703 to Regulate AKT/mTOR Pathway
}

This article was published in the following Dove Press journal:

Cancer Management and Research

\section{Jingyun Guo* \\ Can Luo* \\ Yuqin Yang (1)* \\ Jianyu Dong \\ Zhaoze Guo \\ Jinlamao Yang \\ Huining Lian \\ Changsheng Ye \\ Minfeng Liu}

Breast Center, Department of General Surgery, Nanfang Hospital, Southern Medical University, Guangzhou 5105I5, People's Republic of China

*These authors contributed equally to this work
Correspondence: Changsheng Ye; Minfeng Liu

Breast Center, Department of General Surgery, Nanfang Hospital, Southern

Medical University, 1838 Guangzhou

Avenue North, Guangzhou 510515,

People's Republic of China

Email yechsh2006@126.com;

125556557@qq.com
Background: Large amounts of microRNAs (miRNAs) have been reported to be aberrantly expressed in malignant cancers. MiR-491-5p makes a significant contribution to the inhibition of multiple cancer processes. However, the specific mechanism and function of miR$491-5 p$ and in breast cancer (BC) is still not fully elucidated.

Methods: MiR-491-5p and ZNF-703 expressions or gene transfection effects were identified by RT-qPCR or Western blot in BC tissues or cells. And ZNF-703 expression was monitored through immunohistochemistry method. Cellular function was also confirmed using Transwell assay. Besides, AKT/mTOR pathway-related proteins were analyzed using Western blotting analysis. Moreover, the interplay between miR-491-5p and ZNF-703 was verified through dual-luciferase reporter assay.

Results: miR-491-5p was lowly expressed, ZNF-703 was highly expressed in BC, and miR491-5p with low expression and ZNF-703 with high expression were associated with poor prognosis of $\mathrm{BC}$ patients. Results of cellular function revealed that overexpression of miR491-5p markedly suppressed BC cell migration and invasion, and knockdown of miR-491-5p had the opposite effect. Besides, mechanism research disclosed that miR-491-5p directly could bind to ZNF-703 and downregulate ZNF-703. Moreover, we proved that ZNF-703 could prominently reverse the influences of miR-491-5p on the migration and invasion of BC cells. More importantly, the data revealed that miR-491-5p repressed AKT/mTOR pathway by ZNF-703 in BC cells.

Conclusion: MiR-491-5p prominently suppresses the metastasis of BC cells through ZNF703 to regulate AKT/mTOR pathway, indicating that miR-491-5p and ZNF-703 might be served as the potential therapeutic targets for BC.

Keywords: breast cancer, miR-491-5p, ZNF-703, AKT/mTOR pathway, migration and invasion

\section{Introduction}

$\mathrm{BC}$ is a type of malignant tumor produced by breast epithelial lesions and is the malignant tissue lesion with the highest incidence in female population. ${ }^{1,2}$ According to statistics, there are more than 1.6 million new BC patients, nearly 500,000 deaths in the world every year, and the incidence of the population has an obvious trend of younger age. ${ }^{3}$ At present, there is no effective method to prevent $\mathrm{BC}$, and surgery and drug therapy have been proved to be effective in the 
early stage of $\mathrm{BC} .{ }^{4}$ However, most $\mathrm{BC}$ patients have already developed tumor metastasis at the time of diagnosis, and early diagnosis directly affects the therapy and prognosis of $\mathrm{BC}$ patients. ${ }^{5}$ Therefore, the in-depth study of the molecular mechanism of $\mathrm{BC}$ invasion and metastasis is conducive to the early discovery, early treatment, and prognosis of BC. Meanwhile, finding specific markers related to tumor lesions has also become a crucial research direction in the field of cancer.

MicroRNAs (mRNAs) are a class of non-coding RNAs containing about 22 nucleotides, which can affect protein expression by reducing the stability of mRNA or inhibiting translation, thus affecting certain biological behavior. ${ }^{6}$ Statistically, there are about 1881 human miRNA genes, which have made significant contributions in eukaryotic cell differentiation, growth, apoptosis, metastasis, and other biological processes. ${ }^{7,8}$ Currently, there are also a large number of researches on the role and mechanism of miRNA in BC progression. ${ }^{9,10}$ Studies suggested that miRNA has the roles of tumor suppressor or oncogene in $\mathrm{BC}$ and other tumors, and its aberrant expression may change the carcinogenesis process. ${ }^{11,12}$ Overexpression or downregulation of inhibitory miRNA can result in inhibition or activation of downstream pro-oncogenic signaling pathways, thereby affecting the function of $\mathrm{BC}$ cells. ${ }^{13}$ Due to the differential roles of various miRNAs in $\mathrm{BC}$, highly specific miRNAs might provide reference value for early diagnosis of BC. Further clarification of the mechanism and role of different miRNAs in $\mathrm{BC}$ is of great significance for diagnosis and targeted therapy. MiR-491$5 \mathrm{p}$, as a member of the miRNA family with regulatory oncogenes, can prevent the processes of multiple cancers, including bladder cancer, ${ }^{14}$ non-Small cell lung cancer, ${ }^{15}$ lung adenocarcinoma, ${ }^{16}$ acute lymphoblastic leukemia, ${ }^{17}$ gastric cancer, ${ }^{18}$ and so on. However, the role and mechanism of miR-491-5p in BC progression are completely unclear. Besides, we predicted that ZNF-703 might be a target gene of miR-491-5p through bioinformatics (Targetscan and miRBase) in our pre-experiment. While whether ZNF-703 could participate in miR-491-5p regulating $\mathrm{BC}$ has yet to be reported.

In our study, the abnormal expressions of ZNF-703 and miR-491-5p were certified in human BC tissues and cells. Besides, we investigated the functions of miR-491-5p overexpression or inhibition in the progression of migration and invasion of BC cells. Moreover, we also screened the differential miR-491-5p-regulated genes, and miR491-5p may directly bind to ZNF-703 and downregulate
ZNF-703 in BC. As well, we explored the potential roles of ZNF-703 in the development and progression of BC mediated by miR-491-5p.

\section{Materials and Methods BC Patients and Specimens}

A total of $30 \mathrm{BC}$ and para-carcinoma tissues were collected from August 2018 to January 2019 at Nanfang Hospital. The histopathology of $\mathrm{BC}$ specimens was independently diagnosed by two pathologists. Before collecting, we have obtained the informed consent from every patient. This present research has also been approved by the Ethics Committee of Nanfang Hospital. All specimens were stored in liquid nitrogen until use.

\section{Cell Lines}

Human breast epithelial cells (MCF10A) and 6 BC cells (MDA-MB-231, MCF7, MDA-MB-453, MDA-MB-157, SK-BR-3, and MDA-MB-468) were also obtained from ATCC (Manassas, VA). MDA-MB-231 cells were cultured in Leibovitz's L-15 medium (Gibco); MCF-7 cells were maintained in Eagle's minimum essential medium (MEM, Gibco); MDA-MB-453 and MDA-MB-468 cells were cultured in DMEM (Invitrogen); MDA-MB-157 and SK-BR -3 cells were cultured inRPMI-1640 medium (Sigma). All media were supplemented with fetal bovine serum (FBS) (Sigma Aldrich), and all cells were grown at $37^{\circ} \mathrm{C}$ under $5 \% \mathrm{CO}_{2}$.

\section{Cell Transfection}

The ZNF-703-overexpressed plasmid and control were purchased from Hanbio Biotechnology Co., Ltd. (Shanghai, China). Negative control (NC), ZNF-703 siRNAs (si-ZNF-703), miR-491-5p mimics, mimics NC, miR-491-5p inhibitors, inhibitors NC were purchased from the GenePharma (China). MCF7 cells $\left(1 \times 10^{5}\right.$ cells/well) in a 6-well plate were transfected with miR-491-5p mimics or/and ZNF-703-overexpressed plasmid, MDA-MB-468 cells $\left(1 \times 10^{5}\right.$ cells/well) in a 6 -well plate were transfected with miR-491-5p inhibitors or/and si-ZNF-703 using Lipofectamine 3000 (Invitrogen) for $48 \mathrm{~h}$ in accordance with the manufacturer's instructions.

\section{RT-qPCR Analysis}

Total RNA was extracted from the $\mathrm{BC}$ tissues or the transfected $\mathrm{BC}$ cells in line with the instructions of TRIzol reagent (Invitrogen, USA). PrimeScript RT reagent 
kit (Takara) was applied for the reverse transcription, and the reaction was $20 \mu \mathrm{L}\left(37^{\circ} \mathrm{C}\right.$ for $1 \mathrm{~h}, 70^{\circ} \mathrm{C}$ for $\left.15 \mathrm{~min}\right)$.

RT-qPCR reaction was conducted using SYBR-Green ${ }^{\circledR}$ (Takara) on the ABI 7900 system. All primer sequences are displayed in Table 1.

\section{Western Blot Assay}

Cells or tissues of each group were washed by precooled PBS, and then incorporated into Lysis Buffer (RIPA, Cell Signaling Technology, Cat.no. 9806). After $20 \mathrm{~min}$ at $4^{\circ} \mathrm{C}$, the lysate was centrifuged $\left(4^{\circ} \mathrm{C}, 12,000 \times \mathrm{g}\right.$ for $\left.40 \mathrm{~min}\right)$ and the supernatant was collected. A total of $30 \mu \mathrm{g}$ protein in each group was isolated using 10\% SDS-PAGE, then transferred to PVDF membranes (Millipore). After blocking, the membranes were incubated with anti-ZNF-703 (1:1000, Abcam), anti-p-AKT (1:1000, Abcam), antiAKT (1:1000, Abcam), anti-p-mTOR (1:1000, Abcam), anti-mTOR (1:1000, Abcam), and GAPDH (1:2000, Abcam) at $4^{\circ} \mathrm{C}$ overnight, and then corresponding secondary antibodies (1:2000, Abcam) for $1 \mathrm{~h}$. ECL reagent (Millipore) was adopted to visualize the results.

\section{Immunohistochemistry (IHC) Assay}

ZNF-703 expression in normal breast tissue, paracarcinoma, and BC tissues was determined by using SP method. Pathological paraffin sections were dewaxed by xylene, dehydrated with gradient ethanol, and repaired with antigen in $10 \mathrm{mmol} / \mathrm{L}$ citrate buffer solution for $2 \mathrm{~min}$. The sections were treated with $3 \%$ hydrogen peroxide peroxidase inhibitor to block endogenous peroxidase, incubated with ZNF-70 antibody at $4^{\circ} \mathrm{C}$ overnight, and then horseradish peroxidase (HRP). Next, the sections were developed with $\mathrm{DAB}$, re-stained with hematoxylin, observed, and scored under a microscope. Results were evaluated by two senior pathologists through independent double-blind method.

Table I The Sequences of Primers in RT-qPCR Assay

\begin{tabular}{|l|l|}
\hline ID & Sequence (5'-3') \\
\hline GAPDH & Forward: TGTTCGTCATGGGTGTGAAC \\
GAPDH & Reverse: ATGGCATGGACTGTGGTCAT \\
ZNF-703 & Reverse: ATGCTGACAAGACGGCGGTT \\
ZNF-703 & Forward: CTACTTGCGGCCCTGCAGGA \\
U6 & Forward: CTTCGGCAGCACATATAC \\
U6 & Reverse: GAACGCTTCACGAATTTGC \\
miR-49I-5p & Forward: GGAGTGGGGAACCCTTCC \\
miR-49I-5p & Reverse: GTGCAGGGTCCGAGGT \\
\hline
\end{tabular}

\section{Transwell Assay}

In the cell migration experiment, the treated cells were placed in the upper of the Transwell chamber (Corning Costar Corp., Cambridge, MA, USA). In the cell invasion experiment, cells were placed in the Matrigel (BD Biosciences) precoated upper chamber. The migration and invasion abilities of cells could be reflected by counting the number of cells entering the lower chamber. The cells in the upper compartment were resuspended in serum-free medium for inoculation, and medium in the lower compartment contained $15 \%$ FBD as chemotaxis. Transwell plate was incubated in a $37^{\circ} \mathrm{C}, 5 \% \mathrm{CO}_{2}$ incubator for $36 \mathrm{~h}$. The cells were fixed with $20 \%$ ethanol, stained with $0.2 \%$ crystal violet (Sigma-Aldrich), and photographed.

\section{Dual-Luciferase Reporter Assay}

The wild-type (WT) and mutant (Mut) ZNF-703 including the binding sites were amplified and inserted into psiCHECKTM2 Vector (Promega). The primers were as follows: WT ZNF703-XhoI F:5'-ccgctcgagCCAGCCCTTCCCCACCGG ACTGTGTATTT-3'; WT ZNF703-NotI R:5'-ataagaatgcgge cgcCCTTCCCCGAACGCAGCTAACGAGCT-3'; Mut ZNF703-F: 5'-CCAGCCCTGTTTTCTCGGACTGTGTATT TATTTACTATAATGTTAGCTTACAAGCTGGGA-3'; Mut ZNF703-R: $\quad$ 5'-TCCCAGCTTGTAAGCTAACATTATAG TAAATAAATACACAGTCCGAGAAAACAGGGCTGG-

3'. Cells were co-transfected with $20 \mathrm{ng}$ miR-491-5p mimics, $20 \mathrm{ng}$ miR-491-5p inhibitors or $20 \mathrm{ng} \mathrm{NC}$ and $100 \mathrm{ng}$ Mut ZNF703, 100 ng WT ZNF703 using Lipofectamine 3000 (Invitrogen) for 48 h. Dual-Luciferase Reporter Assay System (Promega) was applied to monitor the luciferase activity.

\section{Statistical Analysis}

All data were expressed as mean \pm standard deviation (SD), and statistical analysis was conducted using SPSS software. Analysis of two or more groups was performed using the Student's $t$-test or Analysis of variance (ANOVA). $P<0.05$ was considered statistically significant.

\section{Results Expression and Prognosis of miR-49I-5p in $B C$}

Based on the Kaplan-Meier Plotter, we analyzed the prognosis of miR-491-5p in BC. And the data from KaplanMeier Plotter exhibited that the BC patients with high expression of miR-491 had a good prognosis relative to 
that with low expression of miR-491-5p ( $P=0.01$, Figure 1A). Besides, the RT-qPCR data disclosed that miR-491$5 \mathrm{p}$ was markedly downregulated in BC tissues $(n=30)$ relative to para-carcinoma tissues $(n=30) \quad(P<0.001$, Figure 1B). These data exhibited that miR-491-5p was lowly expressed in BC, and low expression of miR-491$5 \mathrm{p}$ was associated with poor prognosis.

\section{miR-49I-5p Markedly Suppressed BC Cell Migration and Invasion}

Besides we further verified the expression changes of miR491-5p in BC cells. The data from RT-qPCR displayed that compared to human breast epithelial cells (MCF10A), the level of miR-491-5p was observably reduced in BC cells (especially MCF7 cells); and among 6 BC cells, miR-491$5 \mathrm{p}$ expression was highest in MDA-MB-468 cells $(P<0.001$, Figure 2A). Therefore, we adopted MCF-7 and MDA-MB-468 cells to overexpress and knock-down miR491-5p in subsequent experiments. Subsequently, we also verified the transfections of miR-491-5p mimics or inhibitors in BC through RT-qPCR assay. The results also uncovered that miR-491-5p was prominently upregulated in MCF7 cells transfected with miR-491-5p mimics compared with NC-mimics $(P<0.001$, Figure $2 \mathrm{~B})$. And miR491-5p was significantly downregulated in MDA-MB-468 cells transfected with miR-491-5p inhibitors relative to NC-inhibitors $(P<0.001$, Figure 2C). Next, Transwell assay was adopted to verify the impacts of miR-491-5p on migration and invasion capabilities of MCF7 and MDA-MB-468 cells. The results presented that overexpression of miR-491-5p could result in a prominent reduction in the migration and invasion capabilities of MCF7 cells $(P<0.001$, Figure 2D). And inhibition of miR-491-5p could lead to a remarkable elevation in the migration and invasion abilities of MDA-MB-468 cells $(P<0.001$, Figure 2E).

\section{miR-49I-5p Prominently Repressed the Phosphorylation of AKT/mTOR Pathway in BC Cells}

AKT/mTOR signaling pathway has been confirmed to contribute to the generation, development, and treatment of cancer cells, which also has become a hot spot in cancer research. In our study, the Western blotting results indicated that the levels of p-AKT and p-mTOR were markedly downregulated in miR-491-5p mimics-transfected MCF7 cells, and significantly upregulated in miR-491-5p inhibitors-transfected MDA-MB-468 cells compared with their respective controls $(P<0.01, P<0.001$, Figure 3$)$. Thus, we testified that miR-491-5p could result in a remarkable inhibition of $\mathrm{AKT} / \mathrm{mTOR}$ pathway in $\mathrm{BC}$ cells.

\section{miR-49I-5p Notably Repressed ZNF-703 Expression by Targeting 3'-UTR of ZNF- 703}

Subsequently, the targeted genes that miR-491-5p might regulate were predicted by bioinformatics analysis, and we discovered that ZNF-703 was the most likely target gene of miR-491-5p. We also exhibited the predicted binding sites between miR-491-5p and ZNF-703 (Figure 4A). Besides, the data from the luciferase reporter assay uncovered that overexpression of miR-491-5p significantly
A

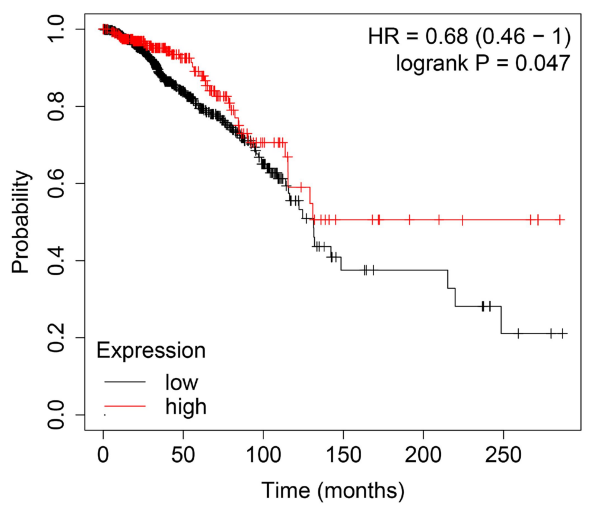

B

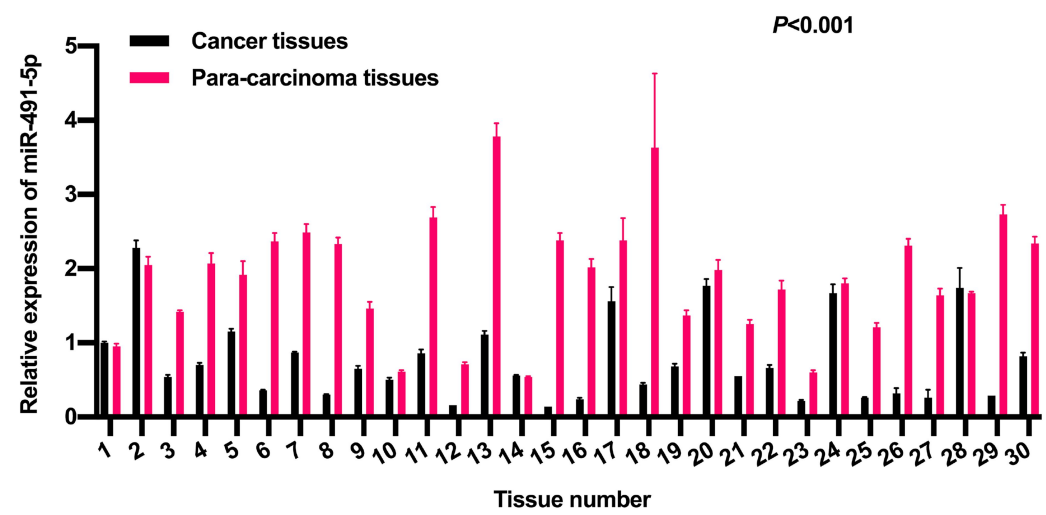

Figure I Expression and prognosis of miR-49I-5p in BC. (A) The prognosis of miR-49I-5p in BC was predicted through the Kaplan-Meier Plotter. (B) RT-qPCR assay was adopted to analyze the level of miR-49I in $B C(n=30)$ and para-carcinoma tissues $(n=30)(P<0.00 I)$. 

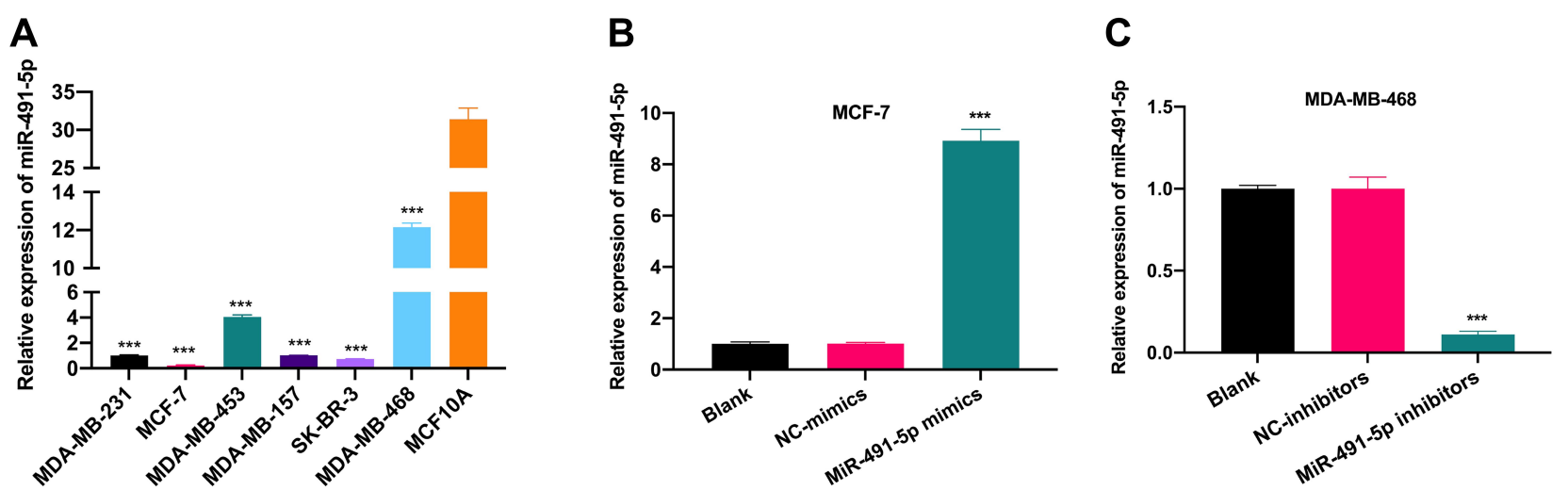

D
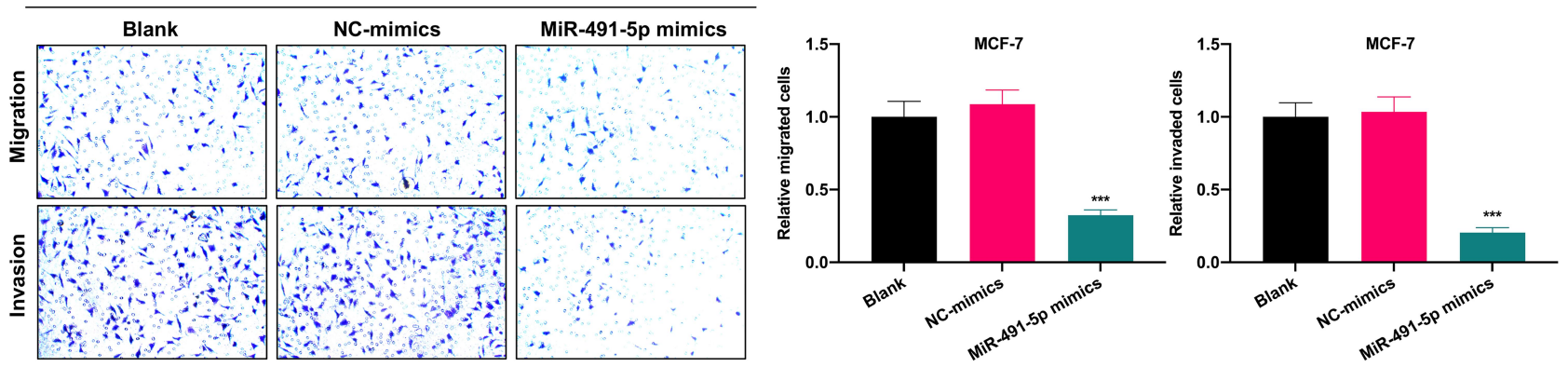

E

MDA-MB-468
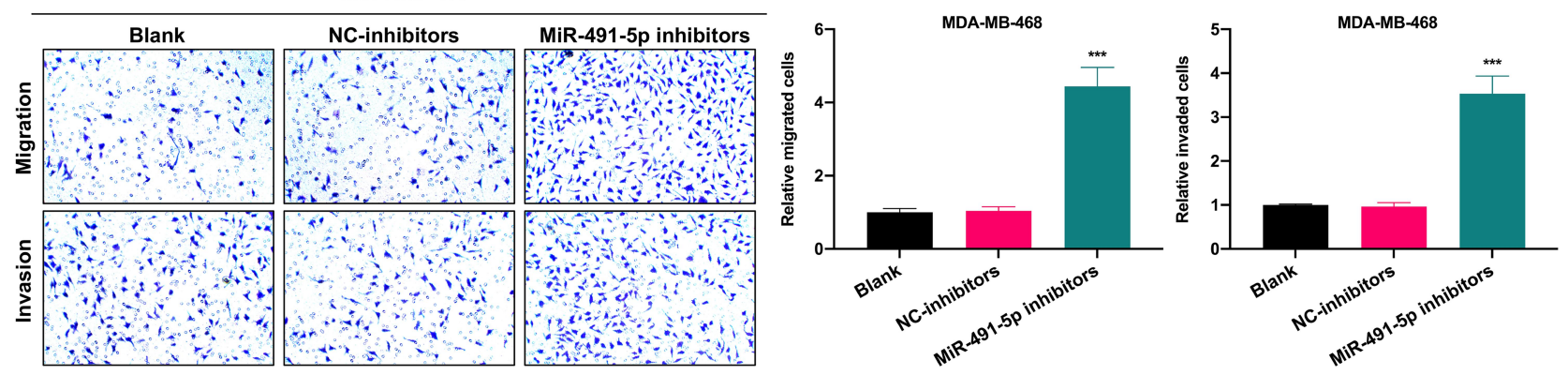

Figure 2 miR-49I-5p markedly suppressed BC cell migration and invasion. (A) Expression of miR-49I-5p was assessed through the usage of RT-qPCR in human breast epithelial cells (MCFIOA) and 6 BC cells (MDA-MB-23I, MCF7, MDA-MB-453, MDA-MB-157, SK-BR-3 and MDA-MB-468). (B) MCF7 cells were transfected with miR-49I$5 p$ mimics or NC-mimics, RT-qPCR analysis was conducted to identify the transfection effect of miR-491-5p mimics in MCF7 cells. (C) MDA-MB-468 cells were transfected with miR-491-5p inhibitors or NC-inhibitors, the transfection effect of miR-49I-5p inhibitors was also confirmed using RT-qPCR in MDA-MB-468 cells. (D) Transwell assay was applied to examine the impacts of miR-49I-5p mimics on the migration and invasion capacities of MCF7 cells. Magnification, $\times 100$. (E) After transfection with miR-49I$5 \mathrm{p}$ inhibitors, the changes in the migration and invasion of MDA-MB-468 cells were also identified by Transwell assay. Magnification, $\times 100$. $* * * P<0.001$.

inhibited the luciferase activity. So, we suggested that miR-491-5p could combine with ZNF-703 mRNA at molecular level $(P<0.05$, Figure 4B). Meanwhile, the results of RT-qPCR and Western blot disclosed that ZNF703 expression was prominently elevated in $6 \mathrm{BC}$ cells relative to MCF10A cells, especially, MCF-7 and SK-RB -3 cells $(P<0.001$, Figure $4 \mathrm{C}$ and D). Moreover, RT-qPCR and Western blot results also testified that overexpression of miR-491-5p observably reduced ZNF-703 expression in MCF-7 cells, inhibition of miR-491-5p signally raised ZNF-703 expression in MDA-MB-468 cells $(P<0.001$, Figure 4E and F). On the whole, we certified that miR- 491-5p dramatically repressed ZNF-703 expression through directly targeting the 3'-UTR of ZNF-703.

\section{ZNF-703 Was Highly Expressed in BC}

Through bioinformatics predictions, we discovered that the BC patients with high expression of ZNF-703 had a poor prognosis relative to that with low expression of ZNF-703 (Figure 5A). The RT-qPCR data exhibited that ZNF-703 was dramatically upregulated in BC tissues $(\mathrm{n}=30)$ relative to para-carcinoma tissues $(\mathrm{n}=30)$ $(P<0.001$, Figure 5B). The Western blot results uncovered that the protein expression level of ZNF-703 was notably 


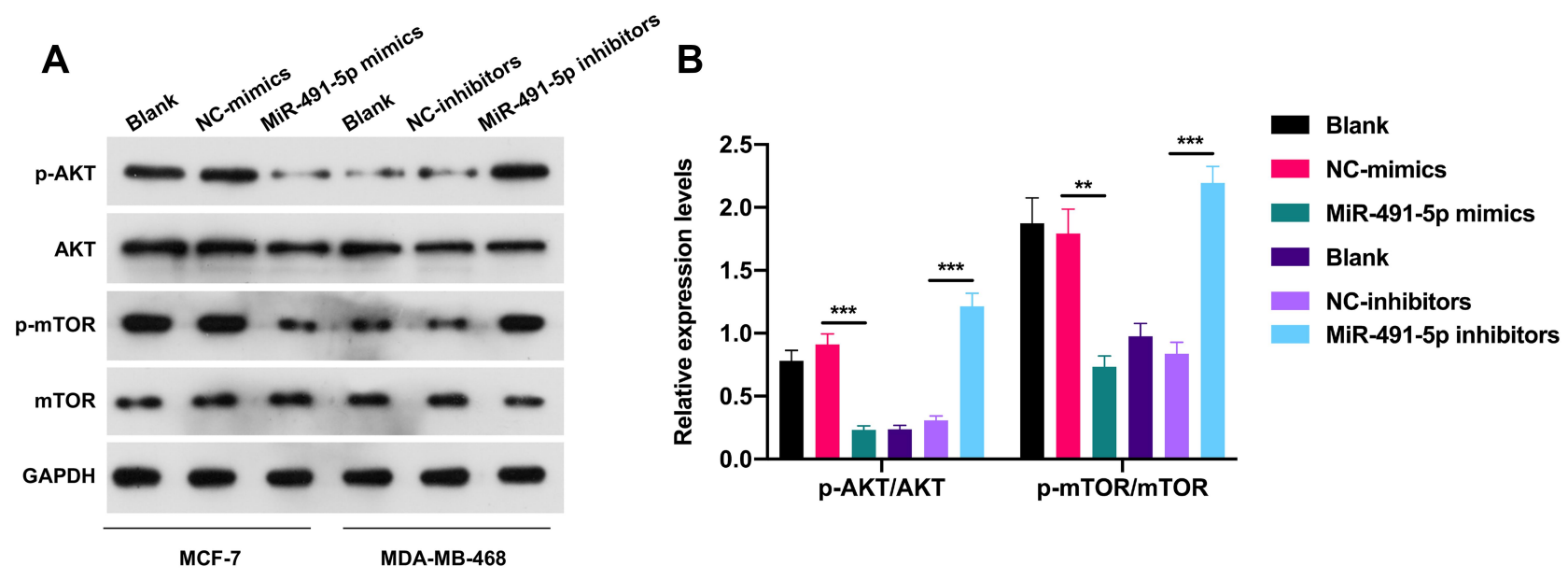

Figure 3 miR-49I-5p prominently repressed the phosphorylation of AKT/mTOR pathway in BC cells. (A) The levels of p-AKT, AKT, p-mTOR and mTOR were certified by Western blotting analysis in miR-49I-5p mimics-transfected MCF7 cells and miR-49I-5p inhibitors-transfected MDA-MB-468 cells. (B) Relative expressions of p-AKT/AKT and $\mathrm{p}-\mathrm{mTOR} / \mathrm{mTOR}$ were calculated using the gray values in Western blot results. $* * P<0.0 \mathrm{I}, * * * p<0.00 \mathrm{I}$.

elevated in $\mathrm{BC}$ tissues with respect to that in paracarcinoma tissues (Figure 5C). Additionally, the IHC results also exhibited that ZNF-703 was signally upregulated in $\mathrm{BC}$ tissues compared with that in para-carcinoma tissues or normal breast tissues (Figure 5D). So, we proved that ZNF-703 was upregulated in BC, and highly expressed ZNF-703 was associated with poor prognosis.

\section{ZNF-703 Prominently Reversed the Inhibitory Effects of miR-49I-5p on the Migration and Invasion of BC Cells}

More importantly, we further investigated whether miR491-5p plays essential roles in migration and invasion of BC cells through its target gene ZNF-703. ZNF-703 was overexpressed using ZNF-703-overexpressed plasmid in MCF-7 cells overexpressing miR-491-5p, and the results further revealed that ZNF-703 expression was memorably increased in miR-491-5p-overexpressed MCF7 cells $(P<0.01, P<0.001$, Figure 6A and C). ZNF703 was silenced using ZNF-703 siRNAs in miR-491-5pinhibited MDA-MB-468 cells, and the data indicated that ZNF-703 expression was dramatically decreased in miR491-5p-inhibited MDA-MB-468 cells $(P<0.001$, Figure 6B and $\mathrm{C}$ ). Next, cellular functional experiment testified that overexpression of ZNF-703 signally enhanced the malignant biological behaviors (migration and invasion) of MCF-7 cells, which have been inhibited by miR-491-5p mimics $(P<0.01, P<0.001$, Figure 6D). Silence of ZNF703 notably prevented the migration and invasion of MDA-MB-468 cells, which have been induced by miR- 491-5p inhibitors $(P<0.05, \quad P<0.01$, Figure 6E). Consequently, we proved that miR-491-5p could memorably prevent the migration and invasion of $\mathrm{BC}$ cells by ZNF-703.

\section{miR-49 I-5p Inhibited the} Phosphorylation of AKT/mTOR Pathway by Targeting ZNF-703 in BC Cells

Likewise, we also explored the impacts of miR-491-5p/ ZNF-703 on AKT/mTOR pathway in BC. As displayed in Figure 7, overexpression of ZNF-703 prominently upregulated p-AKT and p-mTOR in miR-491-5p-overexpressed MCF-7 cells; silence of ZNF-703 significantly downregulated $\mathrm{p}-\mathrm{AKT}$ and $\mathrm{p}-\mathrm{mTOR}$ in miR-491-5p-inhibited MDAMB-468 cells $(P<0.01, P<0.001)$. Hence, we proved that ZNF-703 markedly induced the activation of AKT/mTOR pathway, which was mediated by miR-491-5p in BC cells.

\section{Discussion}

$\mathrm{BC}$ is a familiar female malignancy, and its incidence rate increases year by year. ${ }^{1,19}$ MiRNAs are highly conserved non-coding small molecule single-stranded RNAs that are widely discovered in plants, animals, and viruses. ${ }^{20,21}$ In the present study, we proved that miR-491-5p was expressed at low levels in $\mathrm{BC}$ tissues and cells, and the low expression of miR-491-5p was relevant to the poor prognosis of $\mathrm{BC}$ patients. Besides, we disclosed that overexpression of miR-491-5p had significant inhibitory effects on the migration and invasion of $\mathrm{BC}$ cells, and inhibition of miR-491-5p had the opposite effect from its 

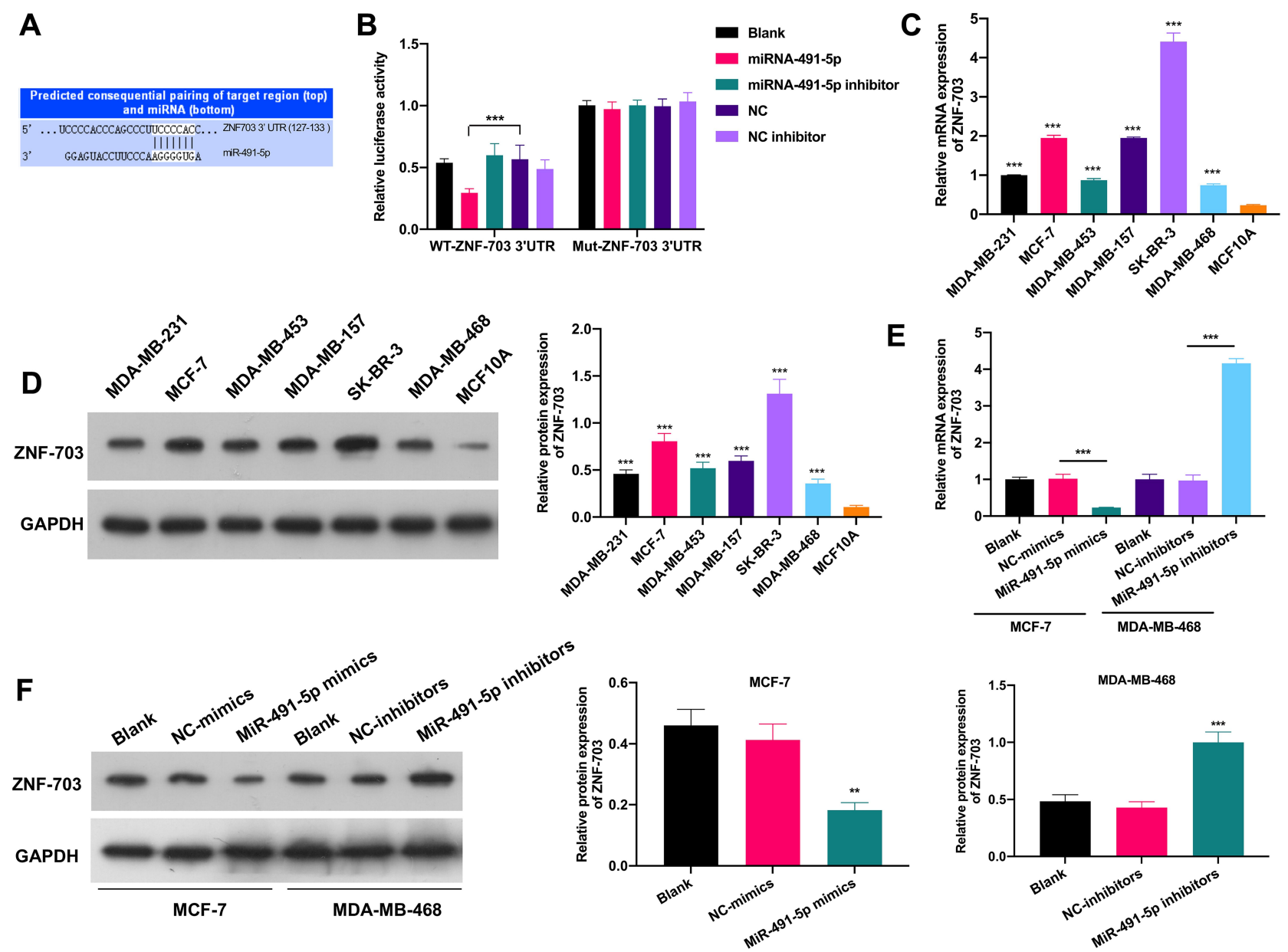

Figure 4 miR-49I-5p notably repressed ZNF-703 expression by targeting 3'-UTR of ZNF-703. (A) Schematic description for the predicted binding sites between miR-49I$5 p$ and ZNF-703. (B) Relative luciferase activity was analyzed via luciferase reporter assay to identify the combination of miR-49I-5p and ZNF-703. ZNF-703 expression was monitored by RT-qPCR (C) and Western blot (D) in MCFIOA and 6 BC cells. (E) ZNF-703 expression was certified through RT-qPCR in miR-49I-5p mimics-transfected MCF7 cells and miR-49I-5p inhibitors-transfected MDA-MB-468 cells. (F) The influence of miR-49I-5p on ZNF-703 expression was monitored by Western blot assay in MCF7 or MDA-MB-468 cells. $* * P<0.01$, $* * * P<0.001$.

overexpression. In recent researches, miR-491-5p also has been reported to be a tumor suppressor gene in a variety of cancers. For instance, lncRNA LBX2-AS1 could memorably induce the proliferation, migration, and invasion of gastric cancer cells by targeting miR-491-5p; ${ }^{18} \operatorname{lncRNA}$ VPS9D1-AS1 could observably accelerate proliferation of acute lymphoblastic leukemia by miR-491-5p; $;^{17}$ Hsa_circ_0001361 could dramatically facilitate invasion and metastasis of bladder cancer cells by miR-491-5p; ${ }^{14}$ IncRNA WDFY3-AS2 could signally enhance the progression of lung adenocarcinoma through miR-491-5p; ${ }^{16}$ LncRNA TTN-AS1 also could markedly boost the progression of non-small cell lung cancer by miR-491-5p. ${ }^{15}$ Therefore, this is the first report about the expression and function of miR-491-5p in BC, while the underlying target genes of miR-491-5p are still unknown in BC.
Zinc finger protein is a specific fingerlike structure protein formed by binding several conserved amino acid residues with zinc ions, which is widely expressed in eukaryotes. ${ }^{22}$ Zinc finger protein is usually applied as a negative transcriptional regulator and participates in vital biological activities such as embryo development, cell differentiation, and gene expression. ${ }^{23,24}$ ZNF703 is a member of the zinc finger protein family and is located in chromosome 8 region (8p11.23). ${ }^{25}$ ZNF703 is a newly discovered breast carcinogen, and with the increase of ZNF703 expression, the survival time of patients decreases and the prognosis becomes worse. ${ }^{26}$ Overexpression of ZNF703 has been reported to be associated with tumor proliferation, differentiation, invasion, and metastasis. ${ }^{27,28}$ Additionally, ZNF703 was verified to be highly expressed in colorectal cancer, ${ }^{29}$ gastric cancer, ${ }^{28}$ head and neck 


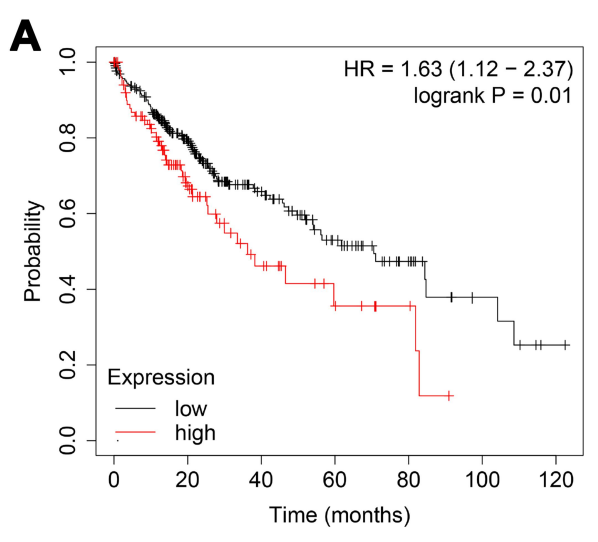

B

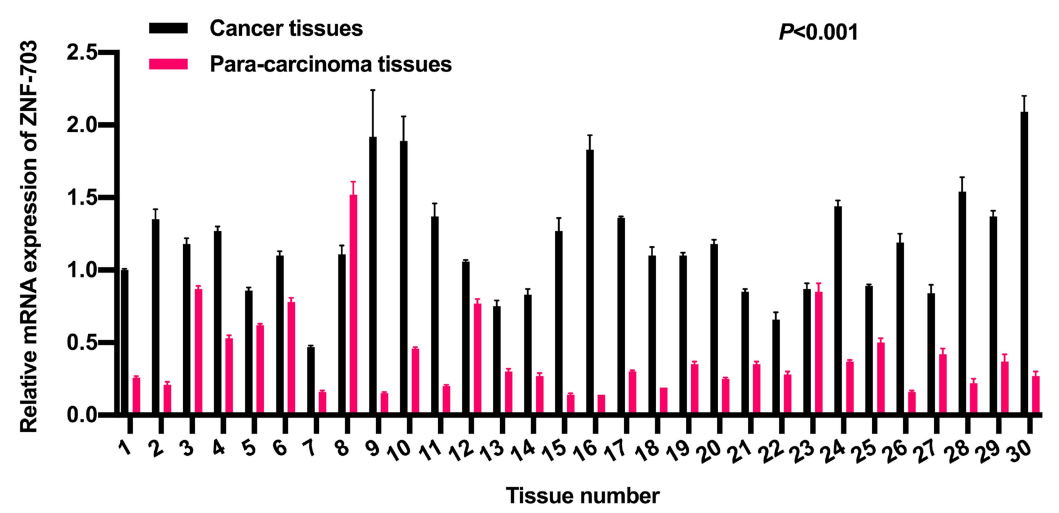

C
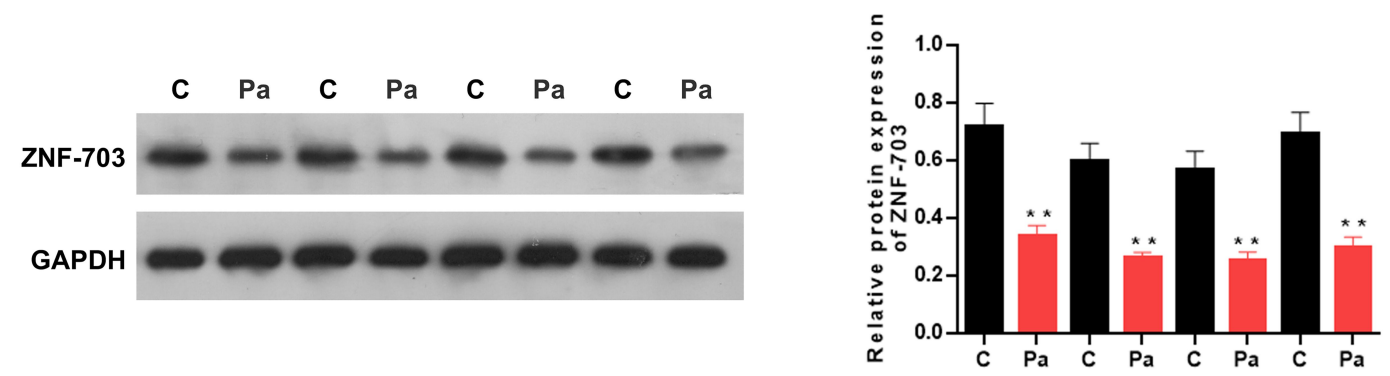

D

Para-carcinoma tissue ( $\times 4$ )

Cancer tissue $(\times 100)$

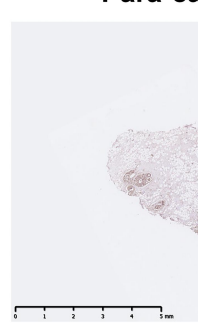

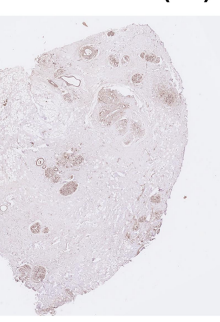
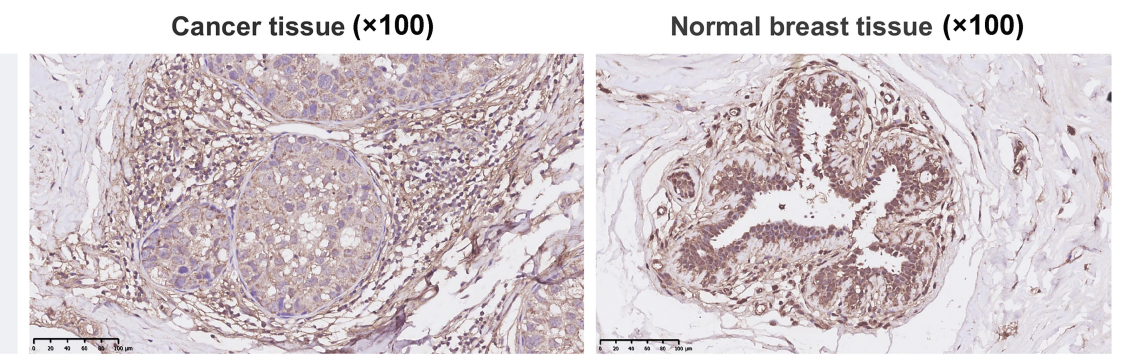

Figure 5 ZNF-703 was highly expressed in BC. (A) The prognosis of ZNF-703 in BC has been presented in the Kaplan-Meier Plotter. (B) The expression of ZNF-703 was examined through RT-qPCR analysis in $B C(n=30)$ and para-carcinoma tissues $(n=30)(P<0.00 I)$. (C) Western blotting analysis of $Z N F-703$ in $B C$ and para-carcinoma tissues, and quantitative analysis of ZNF-703 expression was conducted based on the gray value. (D) IHC assay was applied to determine ZNF-703 expression in BC tissues, paracarcinoma tissues and normal breast tissues. $* * P<0.01$.

squamous cell carcinoma, ${ }^{30}$ etc. In our study, we further revealed that ZNF-703 was highly expressed in BC, and the upregulation of ZNF-703 was associated with poor prognosis of $\mathrm{BC}$ patients. Moreover, we demonstrated that ZNF-703 was a target gene of miR-491-5p, and could be prominently downregulated by miR-491-5p in BC. In cellular functions, we also testified that ZNF703 could prominently recover the inhibitory effects of miR-491-5p on the migration and invasion of BC cells.

AKT/mTOR signaling pathway has significant effects on the occurrence, development, and prognosis of epithelialderived malignancies. ${ }^{31}$ Researches manifested that the abnormal activation of $\mathrm{AKT} / \mathrm{mTOR}$ pathway can result in malignant proliferation, blocked apoptosis, accelerated cycle operation, angiogenesis, invasion and metastasis, etc. ${ }^{32-34}$ Currently, AKT/mTOR pathway has become a novel target for tumor therapy. In our study, we also verified that overexpression of miR-491-5p prominently inhibited the activation of AKT/ mTOR pathway, and inhibition of miR-491-5p induced the activation of $\mathrm{AKT} / \mathrm{mTOR}$ pathway in $\mathrm{BC}$ cells. Besides, the abnormal changes of AKT/mTOR pathway mediated by miR491-5p could also be significantly reversed in BC. Therefore, miR-491-5p repressed metastasis of BC by targeting ZNF-703 to regulate $\mathrm{AKT} / \mathrm{mTOR}$ pathway. In our future research, we will explore the potential functions and mechanisms of miR491-5p/ZNF-703 axis in BC in greater depth and rigor. 
A

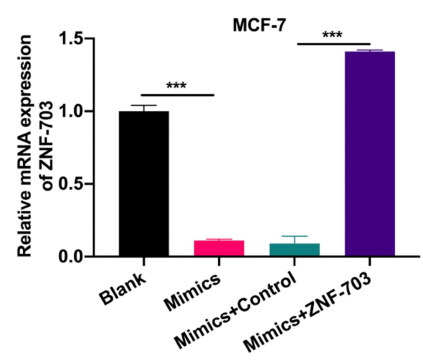

B

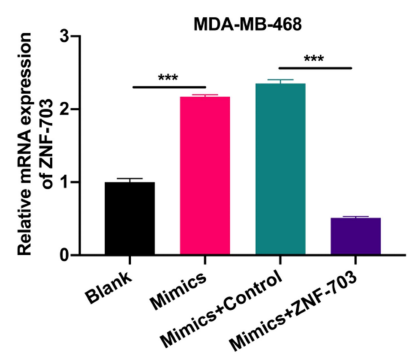

C

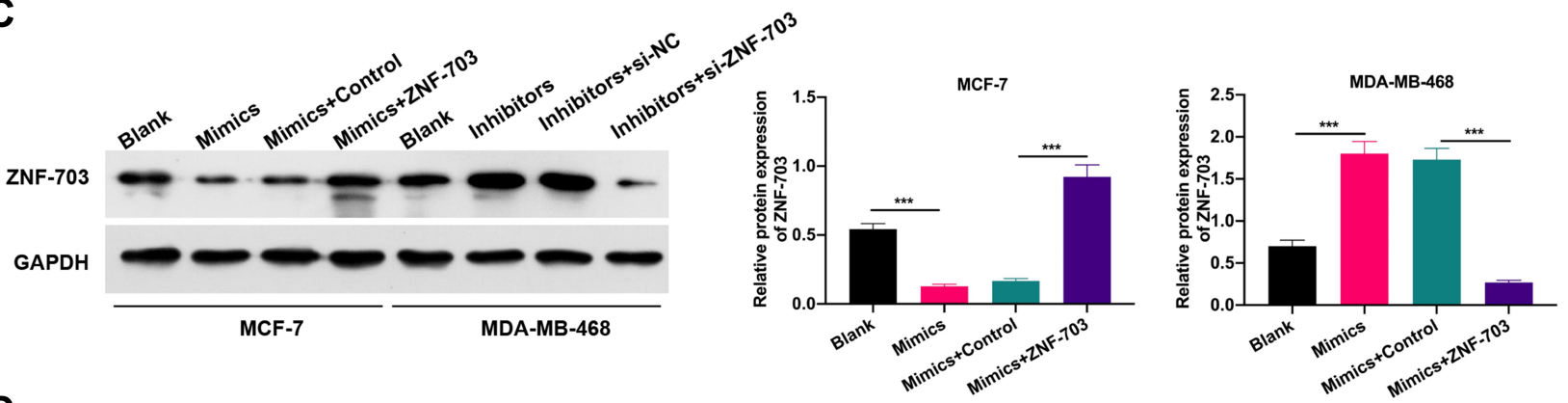

D

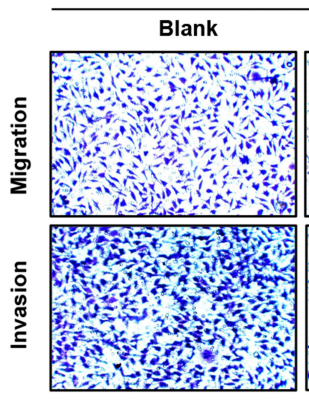

MCF-7

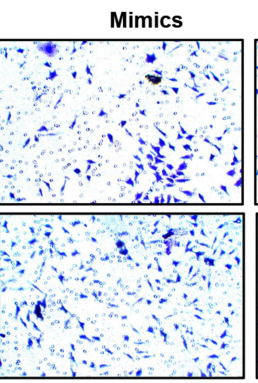

\section{Mimics+Control Mimics+ZNF-703}

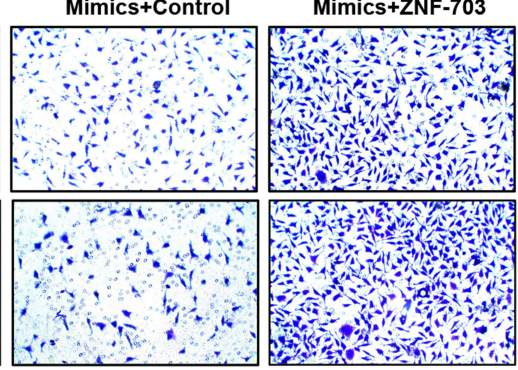

MDA-MB-468

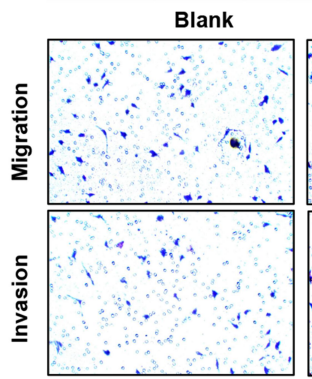

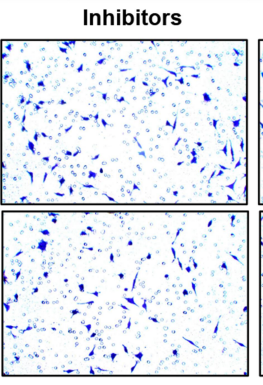

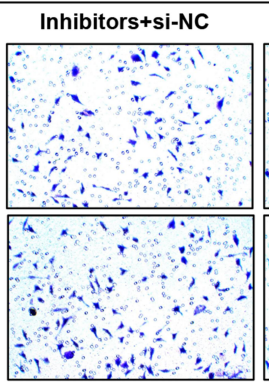

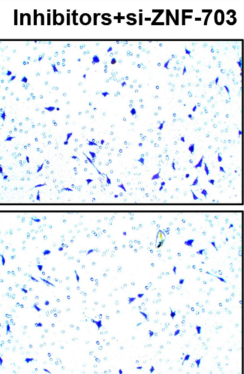

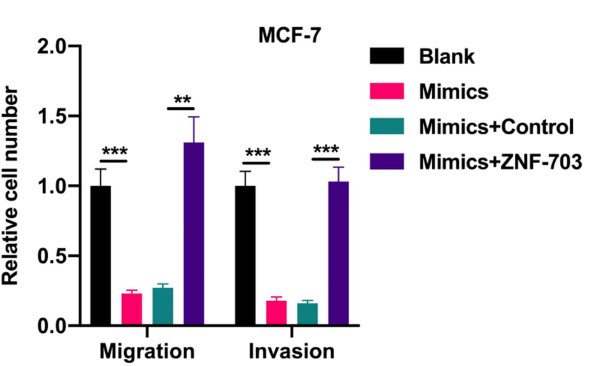

Figure 6 ZNF-703 prominently reversed the inhibitory effects of miR-49I-5p on the migration and invasion of BC cells. (A) miR-49I-5p-overexpressed MCF7 cells were transfected with ZNF-703-overexpressed plasmid or control, and RT-qPCR was conducted to monitor the expression of ZNF-703. (B) ZNF-703 expression was certified through RT-qPCR assay in MDA-MB-468 cells which were transfected with miR-49I-5p inhibitors and si-ZNF-703 or si-NC. (C) Western blotting analysis of ZNF-703 was presented in MCF7 cells transfected with miR-49I-5p mimics and ZNF-703-overexpressed plasmid and MDA-MB-468 cells transfected with miR-49I-5p inhibitors and siZNF-703. (D) After co-transfection with miR-49I-5p mimics and ZNF-703-overexpressed plasmid, the migration and invasion capabilities of MCF7 cells were analyzed via Transwell assay. Magnification, $\times 100$. (E) Transwell assay was also carried out to examine the impacts of miR-49I-5p inhibitors and si-ZNF-703 on the migration and invasion of MDA-MB-468 cells. Magnification, $\times 100$. $* P<0.05, * * P<0.01$, $* * * P<0.001$.

\section{Conclusions}

Current research confirmed that the high expression of miR-491-5p was relevant to the good prognosis and BC pathogenesis by preventing migration and invasion. And miR-491-5p can down-regulate ZNF-703 through targeted binding, and repress the activation of $\mathrm{AKT} / \mathrm{mTOR}$ pathway in BC. More importantly, we revealed ZNF-703 could notably reverse the effects of miR-491-5pin BC cells. Therefore, the study of miR-491-5p/ZNF-703 axis and $\mathrm{AKT} / \mathrm{mTOR}$ pathway in the development of $\mathrm{BC}$ is crucial 


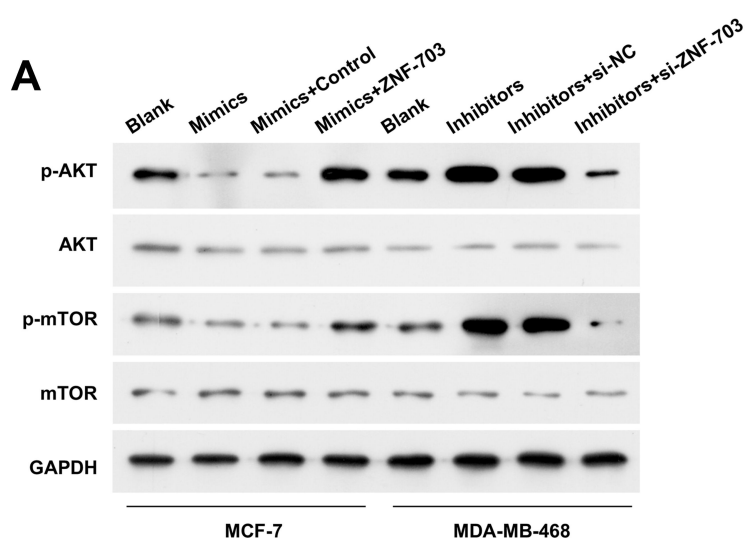

B

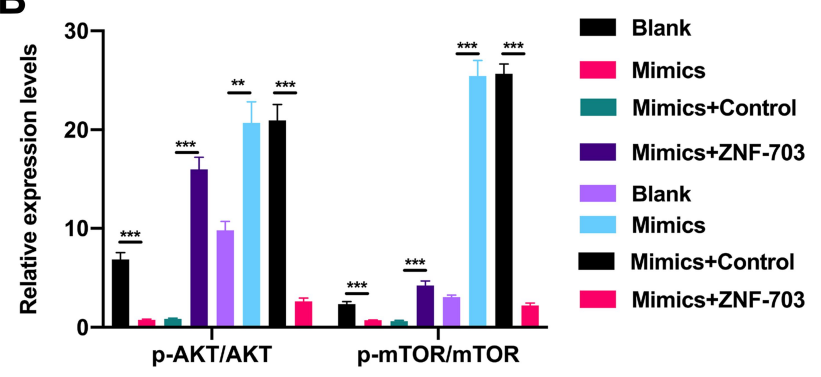

Figure 7 miR-49I-5p inhibited the phosphorylation of AKT/mTOR pathway by targeting ZNF-703 in BC cells. (A) Western blotting analysis of p-AKT, AKT, p-mTOR and mTOR in miR-49I-5p mimics and ZNF-703 co-transfected MCF7 cells, and miR-49I-5p inhibitors and si-ZNF-703 co-transfected MDA-MB-468 cells. (B) Quantitative analysis of $\mathrm{p}-\mathrm{AKT} / \mathrm{AKT}$ and $\mathrm{p}-\mathrm{mTOR} / \mathrm{mTOR}$ was calculated in line with the gray values. ${ }^{*} * P<0.01, * * * P<0.001$.

for the study of targeted therapy drugs with strong specificity and low toxicity in BC.

\section{Funding}

This work was supported by President Foundation of Nanfang Hospital, Southern Medical University (Grant No. 2016L007).

\section{Disclosure}

The authors have declared no competing interests.

\section{References}

1. Pakiž M, Lukman L, Kozar N. Patients' and physicians' expectations differ significantly during the follow-up period after completion of primary treatment of gynecological or breast cancer. Eur J Gynaecol Oncol. 2019;40(5):781-786.

2. Watkins EJ. Overview of breast cancer. JAAPA. 2019;32(10):13-17. doi:10.1097/01.JAA.0000580524.95733.3d

3. Thorat MA, Balasubramanian R. Breast cancer prevention in high-risk women. Best Pract Res Clin Obstet Gynaecol. 2020;65:18-31. doi:10.1016/j.bpobgyn.2019.11.006

4. Rossi L, Mazzara C, Pagani O. Diagnosis and treatment of breast cancer in young women. Curr Treat Options Oncol. 2019;20(12):86. doi:10.1007/s11864-019-0685-7
5. Slepicka PF, Cyrill SL, Dos Santos CO. Pregnancy and breast cancer: pathways to understand risk and prevention. Trends Mol Med. 2019;25(10):866-881. doi:10.1016/j.molmed.2019.06.003

6. Bhaskaran M, Mohan M. MicroRNAs: history, biogenesis, and their evolving role in animal development and disease. Vet Pathol. 2014;51 (4):759-774. doi:10.1177/0300985813502820

7. Lu TX, Rothenberg ME. MicroRNA. J Allergy Clin Immunol. 2018;141(4):1202-1207. doi:10.1016/j.jaci.2017.08.034

8. Saliminejad K, Khorram Khorshid HR, Soleymani Fard S, Ghaffari SH. An overview of microRNAs: biology, functions, therapeutics, and analysis methods. $J$ Cell Physiol. 2019;234 (5):5451-5465. doi:10.1002/jcp. 27486

9. Fridrichova I, Zmetakova I. MicroRNAs contribute to breast cancer invasiveness. Cells. 2019;8(11):1361. doi:10.3390/cells8111361

10. Rahman MM, Brane AC, Tollefsbol TO. MicroRNAs and epigenetics strategies to reverse breast cancer. Cells. 2019;8(10):1214. doi:10.3390/cells 8101214

11. Loh HY, Norman BP, Lai KS, Rahman N, Alitheen NBM, Osman MA. The regulatory role of MicroRNAs in breast cancer. Int J Mol Sci. 2019;20(19):4940. doi:10.3390/ijms20194940

12. Daoud AZ, Mulholland EJ, Cole G, McCarthy HO. MicroRNAs in pancreatic cancer: biomarkers, prognostic, and therapeutic modulators. BMC Cancer. 2019;19(1):1130. doi:10.1186/s12885019-6284-y

13. Boca S, Gulei D, Zimta AA, et al. Nanoscale delivery systems for microRNAs in cancer therapy. Cell Mol Life Sci. 2020;77 (6):1059-1086.

14. Liu F, Zhang H, Xie F, et al. Hsa_circ_0001361 promotes bladder cancer invasion and metastasis through miR-491-5p/MMP9 axis. Oncogene. 2020;39(8):1696-1709. doi:10.1038/s41388-019-1092-z

15. Qi G, Li L. LncRNA TTN-AS1 promotes progression of non-small cell lung cancer via regulating miR-491-5p/ZNF503 axis. Onco Targets Ther. 2020;13:6361-6371. doi:10.2147/OTT.S238890

16. Ren P, Hong X, Chang L, Xing L, Zhang H. USF1-induced overexpression of long noncoding RNA WDFY3-AS2 promotes lung adenocarcinoma progression via targeting miR-491-5p/ZNF703 axis. Mol Carcinog. 2020;59(8):875-885. doi:10.1002/mc.23181

17. Xiao S, Xu N, Ding Q, Huang S, Zha Y, Zhu H. LncRNA VPS9D1-AS1 promotes cell proliferation in acute lymphoblastic leukemia through modulating GPX1 expression by miR-491-5p and miR-214-3p evasion. Biosci Rep. 2020;40(10). doi:10.1042/ BSR20193461

18. Xu G, Zhang Y, Li N, et al. LBX2-AS1 up-regulated by NFIC boosts cell proliferation, migration and invasion in gastric cancer through targeting miR-491-5p/ZNF703. Cancer Cell Int. 2020;20(1):136. doi:10.1186/s12935-020-01207-w

19. Wörmann B. Breast cancer: basics, screening, diagnostics and treatment. Med Monatsschr Pharm. 2017;40(2):55-64.

20. Bushati N, Cohen SM. MicroRNA functions. Annu Rev Cell Dev Biol. 2007;23(1):175-205. doi:10.1146/annurev.cellbio.23.090506.123406

21. Mohr AM, Mott JL. Overview of microRNA biology. Semin Liver Dis. 2015;35(1):3-11. doi:10.1055/s-0034-1397344

22. Laity JH, Lee BM, Wright PE. Zinc finger proteins: new insights into structural and functional diversity. Curr Opin Struct Biol. 2001;11 (1):39-46. doi:10.1016/S0959-440X(00)00167-6

23. Chiu H-W, Chen C-H, Chang J-N, Chen C-H, Hsu Y-H. Far-infrared promotes burn wound healing by suppressing NLRP3 inflammasome caused by enhanced autophagy. J Mol Med. 2016;94(7):809-819. doi:10.1007/s00109-016-1389-0

24. Squassina A, Meloni A, Chillotti C, Pisanu C. Zinc finger proteins in psychiatric disorders and response to psychotropic medications. Psychiatr Genet. 2019;29(5):132-141. doi:10.1097/ YPG.0000000000000231

25. Sircoulomb F, Nicolas N, Ferrari A, et al. ZNF703 gene amplification at 8 p12 specifies luminal B breast cancer. EMBO Mol Med. 2011;3 (3):153-166. doi:10.1002/emmm.201100121 
26. Orhan C, Bakır B, Dalay N, Buyru N. ZNF703 is an important player in head and neck cancer. Clin Otolaryngol. 2019;44(6):1080-1086. doi: $10.1111 /$ coa. 13450

27. Baykara O, Dalay N, Kaynak K, Buyru N. ZNF703 overexpression may act as an oncogene in non-small cell lung cancer. Cancer Med. 2016;5(10):2873-2878. doi:10.1002/cam4.847

28. Yang G, Ma F, Zhong M, et al. ZNF703 acts as an oncogene that promotes progression in gastric cancer. Oncol Rep. 2014;31 (4):1877-1882. doi:10.3892/or.2014.2997

29. Ma F, Bi L, Yang G, et al. ZNF703 promotes tumor cell proliferation and invasion and predicts poor prognosis in patients with colorectal cancer. Oncol Rep. 2014;32(3):1071-1077. doi:10.3892/or.2014.3313

30. Yang H, Jiang WQ, Cao Y, et al. Elevated ZNF703 protein expression is an independent unfavorable prognostic factor for survival of the patients with head and neck squamous cell carcinoma. Dis Markers. 2015;2015:640263. doi:10.1155/2015/640263
31. Aoki M, Fujishita T. Oncogenic roles of the PI3K/AKT/mTOR axis Curr Top Microbiol Immunol. 2017;407:153-189. doi:10.1007/ $82 \_2017 \quad 6$

32. Ghoneum A, Said N. PI3K-AKT-mTOR and NFאB pathways in ovarian cancer: implications for targeted therapeutics. Cancers (Basel). 2019;11(7):949. doi:10.3390/cancers11070949

33. Rahmani F, Ziaeemehr A, Shahidsales S, et al. Role of regulatory miRNAs of the PI3K/AKT/mTOR signaling in the pathogenesis of hepatocellular carcinoma. J Cell Physiol. 2020;235(5):4146-4152. doi:10.1002/jcp. 29333

34. Sharma V, Sharma AK, Punj V, Priya P. Recent nanotechnological interventions targeting PI3K/Akt/mTOR pathway: a focus on breast cancer. Semin Cancer Biol. 2019;59:133-146. doi:10.1016/j. semcancer.2019.08.005

\section{Publish your work in this journal}

Cancer Management and Research is an international, peer-reviewed open access journal focusing on cancer research and the optimal use of preventative and integrated treatment interventions to achieve improved outcomes, enhanced survival and quality of life for the cancer patient.
The manuscript management system is completely online and includes a very quick and fair peer-review system, which is all easy to use. Visit http://www.dovepress.com/testimonials.php to read real quotes from published authors. 